This item was submitted to Loughborough's Research Repository by the author.

Items in Figshare are protected by copyright, with all rights reserved, unless otherwise indicated.

\title{
Conceptual engineering for mathematical concepts
}

PLEASE CITE THE PUBLISHED VERSION

https://doi.org/10.1080/0020174X.2017.1385526

PUBLISHER

(c) Taylor \& Francis

VERSION

AM (Accepted Manuscript)

PUBLISHER STATEMENT

This work is made available according to the conditions of the Creative Commons Attribution-NonCommercialNoDerivatives 4.0 International (CC BY-NC-ND 4.0) licence. Full details of this licence are available at: https://creativecommons.org/licenses/by-nc-nd/4.0/

\section{LICENCE}

CC BY-NC-ND 4.0

\section{REPOSITORY RECORD}

Tanswell, Fenner. 2019. "Conceptual Engineering for Mathematical Concepts”. figshare. https://hdl.handle.net/2134/34935. 


\title{
Conceptual Engineering for Mathematical Concepts
}

\author{
Fenner Stanley Tanswell \\ Department of Computer Science \\ University of Oxford \\ Wolfson Building, Keble Road \\ Oxford, Oxfordshire, UK OX1 3QD. \\ Forthcoming in Inquiry in a special issue on \\ Ameliorative Projects and Conceptual Engineering.
}




\begin{abstract}
In this paper I investigate how conceptual engineering applies to mathematical concepts in particular. I begin with a discussion of Waismann's notion of open texture, and compare it to Shapiro's modern usage of the term. Next I set out the position taken by Lakatos which sees mathematical concepts as dynamic and open to improvement and development, arguing that Waismann's open texture applies to mathematical concepts too. With the perspective of mathematics as open-textured, I make the case that this allows us to deploy the tools of conceptual engineering in mathematics. I will examine Cappelen's recent argument that there are no conceptual safe spaces and consider whether mathematics constitutes a counterexample. I argue that it does not, drawing on Haslanger's distinction between manifest and operative concepts, and applying this in a novel way to set-theoretic foundations. I then set out some of the questions that need to be engaged with to establish mathematics as involving a kind of conceptual engineering. I finish with a case study of how the tools of conceptual engineering will give us a way to progress in the debate between advocates of the Universe view and the Multiverse view in set theory.
\end{abstract}

Keywords: Conceptual Engineering, Mathematical Concepts, Open Texture, Mathematical Practice, Lakatos 


\section{Introduction}

Mathematical concepts are of great relevance to the general process and methodology of conceptual engineering. Conversely, conceptual engineering gives us useful theoretical tools and insights into the practice of mathematics. In this paper I will be exploring both of these directions, setting out how mathematicians can be seen as a kind of conceptual engineers and how looking at mathematical concepts in particular can illustrate and help us to evaluate arguments and distinctions deployed in conceptual engineering, especially by (Cappelen forthcoming) and (Haslanger 2012).

While the central examples of conceptual engineering in the literature have received substantial attention recently, the realm of mathematics has only been lightly touched upon, such as in (Scharp \& Shapiro forthcoming) and implicitly in the work of Øystein Linnebo (Linnebo 2016). This is not to say that the development and change of mathematical concepts has not been considered more generally outside of the discussions of conceptual engineering; for example, the work of Lakatos on this subject is widely known (Lakatos 1976), something I will be discussing below. Nonetheless, there is a temptation to view mathematics in an idealised way as a safe-haven for concepts, where rigour and formality protect against the defects, imprecision, sloppiness and inconsistencies of everyday concepts. I will argue that we would be wrong to fully give in to this temptation, and that mathematics is not a safe space where we are in strict control of our concepts. Mathematics depends on the informality and creativity in the concepts being used for it, and as a result mathematics also involves a great deal of choice, development and engineering of the concepts involved.

I will begin in section 2 with a more general discussion of the importance of Waismann's notion of open texture. While conceptual engineering commonly refers back to Carnapian explication as its modern forerunner, I will show that open texture is equally relevant and provides a useful way to think about the fixity of concepts. I also contrast Waismann's original description of open texture with Shapiro's recent discussion of it, which is also of independent interest. Following Shapiro, in section 3 I will demonstrate that a major lesson to be learned from Lakatos is that mathematical concepts can be open textured in Waismann's sense. Next, in section 4, I will argue that mathematics is not a conceptual safe space in the terminology of (Cappelen forthcoming), arguing from the open texture of mathematical concepts and the problem of semantic contamination in mathematics. Following up on this, in section 5 I will deploy Haslanger's manifest/operative concept distinction to the case of set theory, showing both that this arises in the supposedly safe space of mathematics and that the tools of conceptual engineering are enlightening when applied to mathematics. In section 6, I will propose that there is serious scope for undertaking a project of conceptual engineering for mathematical concepts and consider the key questions that should be addressed as part of it. Finally, in section 7 I will show how these questions will apply to the case of the contemporary debate between the Universe and Multiverse views of set theory. This will demonstrate that importing the conceptual engineering perspective into mathematics will 
suggest a novel and exciting new way to address a difficult and controversial issue in the foundations of mathematics. ${ }^{1}$

\section{Waismann and Open Texture}

Let us begin by investigating what it is for a concept to display open texture, as it is used by Waismann in (Waismann 1968) and Shapiro in (Shapiro 2006) ${ }^{2}$ It should be noted that although Shapiro follows Waismann in general, the exact characterisation of open texture does shift somewhat between them. I shall set out the two definitions and see how closely they agree. The main difference between the two seems to come down to the difference between the potential for sharpening concepts versus the potential for extending the domain of the concepts.

Waismann introduces the term 'open texture' through examples such as the following regarding our concept of cat:

What, for instance, should I say when that creature later on grew to gigantic size? Or if it showed some queer behaviour usually not to be found with cats, say, if, under certain conditions, it could be revived from death whereas normal cats could not? Shall I, in such a case, say that a new species has come into being? Or that it was a cat with extraordinary properties? (Waismann 1968, p. 119)

Further examples include people who show the unusual feature of disappearing and re-appearing, someone who is old enough to remember King Darius and a lump of gold which emits a new kind of radiation. The point is that in such surprising situations the concepts we possess do not settle whether these are just unusual manifestations of the familiar concept or the appearance of some new thing entirely. Waismann links this up to two crucial points: (1) that our concepts are only delimited in some possible directions and not others, and (2) what he calls "the essential incompleteness of an empirical description" (Waismann 1968, p. 121).

Waismann never gives an explicit definition of open texture ${ }^{3}$, but the relevant property found in the examples appears to be that of (1): that the concepts we deploy and use to understand the world around us are not delimited in all

\footnotetext{
${ }^{1}$ Something to note in the following is that I am unashamedly glossing over whether the central point is one about concepts or about our use of language. This is not because it is unimportant in general (it definitely is important) but because I don't think anything I have to say particularly depends on it. I am drawing on a number of authors, who seem to go different ways on the question, and constantly changing terminology would be cumbersome for little payoff in this setting.

${ }^{2}$ Shapiro's book has an appendix focussed entirely on Waismann's account of open texture (Shapiro 2006, pp. 210-215).

${ }^{3}$ It may be that open texture itself is the sort of thing that avoids full specification, such that the concept of open texture is itself open-textured. Such considerations are familiar from the literature on vagueness. The connection between open texture and vagueness will be discussed shortly.
} 
possible ways. Indeed, let us take this as the central component of Waismann's account of open texture to give the following definition:

Open Texture 1 (OT1) A concept or term displays open texture iff there are possible objects falling outside of the standard domain of application for which there is no fact of the matter as to whether they fall under the concept or not.

In other words, Waismann's idea of open texture concerns the potential to expand concepts to new or larger domains. Concepts generally do have a something approaching a standard domain of application, a range of practical situations in which we know which objects do and do not fall under that concept. However, these normal domains do not cover all potential applications and his examples provide cases where we are asked to apply the concepts beyond the standard situations, and there is no fact of the matter as to whether the objects in question should fall under the relevant concepts or not. Shapiro puts it as follows:

We language users introduce terms to apply to certain objects or kinds of objects, and, of course, the terms are supposed to fail to apply to certain objects or kinds of objects. As we introduce the terms, and use them in practice, we cannot be sure that every possible situation is covered. (Shapiro 2006, p. 210)

A good way to think about this is presented by Bartha in terms of hard and easy cases:

Typically, the decision about whether the predicate applies involves a nontrivial comparison to paradigm cases or prototypes. There will be "easy cases" where there is general agreement that the predicate does or does not apply, and "hard cases" where applicability is open to debate. (Bartha 2010, p. 9)

Importantly, open texture does not require that we have already identified such areas of openness, only that it is possible that there are such areas. The mere potential for new situations to arise in which we can question how the concepts are applied is enough. We do not need to know in advance which kinds of questions will push us outside of the standard domain of application, and in fact we usually don't know this in advance and usually don't predict the difficult situations that might arise for our application of concepts.

Shapiro's version of open texture is slightly different. In (Shapiro 2006), Shapiro is arguing for an account of vagueness which rests on open texture. However, we can observe that the definition he uses is not quite the same:

Suppose, again, that $a$ is a borderline case of $P$. I take it as another premise that, in at least some situations, a speaker is free to assert $P a$ and free to assert $\neg P a$, without offending against the meanings of the terms, or against any other rule of language use. Unsettled 
entails open. The rules of language use, as they are fixed by what we say and do, allow someone to go either way. Let us call this the open-texture thesis. (Shapiro 2006, p. 10)

Let us take this characterisation to be definitional of open texture in the sense he uses it:

Open Texture 2 (OT2) A concept or term displays open texture iff there are cases for which a competent, rational agent may acceptably assert either that the concept applies or that it disapplies.

While Waismann's account of open texture is about the extent to which a concept is delimited, in contrast Shapiro's definition is about competent language users being able to go either way on whether or not a term should be applied or a proposition asserted. What seems to be driving this definition in the text, though, is that the open texture in OT2 will be applicable to borderline cases, thus also includes the potential for competent agents to go about deciding one way or another and sharpening the concepts. The difference becomes less marked, however, if we realise that on Waismann's account, in going beyond a concept's standard domain a competent speaker can go either way on the application of the term corresponding to the open-textured concept, as we have on Shapiro's definition.

The main difference between the two definitions is with respect to vagueness, through which we see that Shapiro's notion is somewhat broader in a certain sense. On Shapiro's account borderline cases of vague terms can count as opentextured because we can sometimes go either way on whether the term applies or not, while on Waismann's definition these will still fall within the standard domain of application (even if they might be hard to decide) so wouldn't count as cases of open texture. For example, taking a paradigm case of vagueness such as bald, there will be some borderline cases in which it isn't clear whether the head in question is bald or not, satisfying the second definition, nonetheless if the person whose hairline is under discussion is a normal one then this will be within the standard domain of application and disapplication of the term 'bald' so the first definition will not be satisfied. However, the term 'bald' will still be open-textured on the first definition because of the possibility of other hard cases not previously considered, say a two-headed person-maybe where one head has no hair and the other has some - how do we apply a term like 'bald' then? An interesting upshot, then, will be that the two definitions seem to agree on the extension of which concepts count as open-textured, because any concepts which permit the vagueness Shapiro is interested in where competent users are allowed to settle borderline cases either way (thereby satisfying OT2) will happen to have cases outwith their standard domain of application for which there is no fact of the matter (thus also satisfying OT1) by the ubiquity of the latter phenomenon. Similarly, if there is no fact of the matter regarding these non-standard cases, it would be strange to insist that a rational agent could not be free to settle it either way if we do choose to extend the cases in this 
direction. $^{4}$

Nonetheless, there is clearly a difference in the kind of phenomenon the two notions are intended to capture. Shapiro's definition is aimed at the broader idea which incorporates both open texture in Waismann's sense and vagueness, by focusing on the openness in the agent's settling of the hard cases. In contrast, Waismann is more concerned with the openness of new cases and potential applications which the concepts might be put to. Indeed, Waismann does compare open texture to vagueness, describing open texture as "something like the possibility of vagueness" (Waismann 1968, p. 120), which is explained as follows:

.... term like 'gold', though its actual use may not be vague, is nonexhaustive or of an open texture in that we can never fill up all the possible gaps through which a doubt may seep in. (Waismann 1968, p. 120)

As Shapiro points out (Shapiro 2006, p. 211), Waismann does not say much more concerning vagueness and certainly doesn't offer an account. However, I believe that I have here identified the source of the differences in the two definitions and their intended phenomena: Shapiro is interested in the sharpening of terms, with the openness to decide either way, while Waismann is concerned about how our terms apply to entirely new cases which might arise.

As a final component in setting out the nature of open texture and Waismann's definition of it, let us return to point (2) left aside above, concerning the "the essential incompleteness of an empirical description". Indeed, I think this will be useful in elaborating on what is meant by a domain of application. The main idea concerns how we describe and define our concepts or terms ${ }^{5}$, where Waismann says:

A term is defined when the sort of situation is described in which it is to be used. (Waismann 1968, p. 122)

Waismann's idea is that the difference between complete and incomplete descriptions and definitions is crucial. A complete description exhausts all the details of its subject and a complete definition "anticipates and settles once for all every possible question of usage" (Waismann 1968, p. 122). Conversely, an incomplete description can be extended with more details that haven't previously been mentioned and an incomplete definition fails to anticipate and settle usage. Waismann's point is that most descriptions of things we find in the world

\footnotetext{
${ }^{4}$ Although it might well be that there are other demands on a rational agent which push them one way or the other, changing the picture I am sketching here. For example, one choice might be rationally preferable over another to remain consistent with the way other concepts have been settled.

${ }^{5}$ Again, one thing I have not discussed is the difference between a concept and a term, i.e. whether these are meant to be mental, linguistic or something else. Clearly, Shapiro's notion leans far more towards the linguistic side and thus towards ascribing open texture to terms. Waismann, however, was unsurprisingly less clear on this matter, switching between concept-talk and term-talk. This is not something I intend to resolve here, nor do I think much hangs on it for my discussion.
} 
will be incomplete, but even further this incompleteness will not be settled as we add further details:

$[\mathrm{H}]$ owever far I go, I shall never reach a point where my description will be completed: logically speaking, it is always possible to extend my description by adding some detail or other. Every description stretches, as it were, into a horizon of open possibilities: however far I go, I shall always carry this horizon with me. (Waismann 1968, p. 122)

Similarly, according to Waismann a definition will not be able to anticipate all eventualities meaning "the process of defining and refining an idea will go on without ever reaching a final stage" (Waismann 1968, p. 123). A standard domain of application and disapplication, then, might just pick out those cases which a definition does anticipate and straightforwardly applies to, which is just to say: Bartha's "easy cases".

To finish, given that we are going to be investigating mathematical concepts, it is interesting to see that Waismann's main comparison for the essentially incomplete empirical terms are the concepts found in mathematics. For example, he takes the description of a triangle by giving the side lengths to be complete and discusses enumerating all possible situations in chess to leave no room for new possibilities to emerge. Put explicitly:

Goldbach's hypothesis [...] may be undecidable [...] But this in no way detracts from the closed texture of the mathematical concepts. If there is no such thing as the (always present) possibility of the emergence of something new, there could be nothing like the open texture of concepts. (Waismann 1968, pp. 123-124)

But I believe Waismann is wrong on both the claims that mathematical concepts are closed-textured and that there is no possibility in the mathematical case of new possibilities arising. Let us turn to Lakatos to see how this might be the case.

\section{Lakatos and the Open Texture of Mathemat- ical Concepts}

In Lakatos's well-known Proofs \& Refutations (Lakatos 1976) he presents a classroom dialogue about various proofs of Euler's formula, the claim that for polyhedra the following relationship holds between the number of vertices (V), edges (E) and faces $(\mathrm{F}): V-E+F=2$. After an initial proof of the claim is proposed, the students begin to find flaws in the proof and propose a whole menagerie of counterexamples, both to the general claim (known as global counterexamples) and to specific lemmas (giving local counterexamples). For example, the "picture frame" is a cuboid with a tunnel through the centre which does not satisfy the equation, and the "twin tetrahedra" are two tetrahedra joined together along a single edge which also falsifies the formula. 
Rather than spelling the end of the theorem, though, the counterexamples reveal deep ambiguities and limitations in the concepts of polyhedron, face, edge and vertex. The dialogue proceeds through various defences of the theorem to rule out difficult counterexamples or to repair the proof in response to them, requiring ever more ingenious maneouvering and mathematical maturity. For example, the best know response is that of "monster-barring" which simply denies that the counterexamples fall under the concept of polyhedron at all, and offers new definitions which exclude the monstrous examples. One of the great joys of the book is that the footnotes reveal that many lines of dialogue are direct quotations from great mathematicians, and that the dialogue itself is a "rational reconstruction" of the history of Euler's formula and the development of the relevant concepts.

Let us now connect Lakatos's ideas to Waismann's notion of open texture, a case also made by Shapiro in (Shapiro 2006, 2013). Recall that Waismann stated that mathematical concepts display closed texture, in contrast with empirical concepts which are open-textured. The aim now is to apply Lakatos's ideas concerning conceptual development in mathematics to show that mathematical concepts can display open texture after all. As Shapiro puts it:

$[T]$ he notion of polyhedron exhibited what Waismann calls opentexture. This open-texture did not prevent mathematicians from working with the notion, and proving things about polyhedra. Still, at the time, it simply was not determinate whether a picture frame counts as a polyhedron. (Shapiro 2006, p. 432)

The point is that the dialogue gives an extensive case study of a mathematical concept which is not fully fixed and delimited. A major piece of Lakatosian terminology is that of heuristic counterexamples: examples which fall outside of the domain of application and disapplication of a concept as it stands, but show that these are too narrow because there are interesting and pertinent cases which have not been covered by the accepted definitions. The first definition of open texture in OT1 covers this same idea, so the existence of heuristic counterexamples with respect to some concept in mathematics - as seen for the concept of polyhedron - is enough to show that that concept displays open texture. The second definition in OT2, concerns the freedom rational agents have to go either way on the application of the term. With respect to the heuristic counterexamples there does seem to be a rational freedom to choose different responses, as demonstrated by the characters in the dialogue. ${ }^{6}$

Waismann takes open texture of concepts to be the result of a lack of full delimitation and definition of when a concept or term applies and disapplies. The reason he takes this to hold for empirical concepts and not mathematical concepts boils down to this. On the one side, Waismann sees empirical descriptions as never being able to anticipate all potential cases and possibilities that might arise concerning their applications. Recall:

\footnotetext{
${ }^{6}$ Though, as we will soon see in the next section, it is not clear how much control we have over the outcomes of these choices. As above, there may be other demands on the decisions made by agents, such as theoretical unity or what leads to the most interesting mathematics.
} 
Every description stretches, as it were, into a horizon of open possibilities: however far I go, I shall always carry this horizon with me.

(Waismann 1968, p. 122)

On the other side, Waismann suggests that this kind of endless possibility of new cases arising is not applicable to mathematics, with its strict, explicit definitions. But this is where he is mistaken, according to the Lakatosian perspective. If one restricts oneself to strict, explicit definitions and ignores the development of mathematical concepts, ideas and theories then we may be convinced about the closed texture of mathematical concepts. However, Lakatos argues that there are ongoing developments and discoveries which are driven by reasons internal to mathematics and that these are made mysterious if mathematics is thought of in this closed-textured way. Rather, the discovery of new mathematics and the maturing of its concepts is bound up with a degree of openness.

There is an important related insight alluded to above by Shapiro: that open texture does not necessarily impede mathematics, but can actually be a healthy part of its development. The closed-texture picture does not give us any insight into how new concepts are introduced, or even how our existing ones are generalised, expanded, adapted etc. On the other hand, acknowledging that the concepts of mathematics are open-textured is theoretically helpful in talking about the development of mathematical ideas. Nonetheless, the open texture of mathematical concepts still allows that in particular domains the concepts are stable enough for them to be used coherently, such that the deductive nature of mathematics is not thrown into turmoil by constant shifts in meaning.

Shapiro also brings out the connection between Waismann and Lakatos concerning the questions surrounding the identity of concepts. In the dialogue we find one character say:

The impact of proofs and refutations on naive concepts is much more revolutionary than that: they erase the crucial naive concepts completely and replace them by proof-generated concepts. (Lakatos 1976, pp. 89-90)

There are then two possibilities: either that the concepts are replaced with entirely different concepts, or else that they are revised, developed and extended in a way that is consistent with them being fundamentally the same concept. The suggestion in this quote that mathematics involves repeated replacement of concepts fits poorly with the picture I outlined in the previous paragraph, although the dialogue form leaves it somewhat unclear whether this quote is Lakatos's view on the matter. Shapiro believes that the Waismannian answers to these questions are best:

I submit that the wise course is to follow Waismann and suggest that the very question of what, exactly, goes into the original, pretheoretic notions is to operate with "too blurred" an expression. One can perhaps claim, now, that the final, austere set-theoretic definition of "polyhedron" [...] is not subject to open texture. Its 
boundaries are as determinate as one could wish [...] But this is not to say that the original, pre-theoretic notion of "polyhedron" was similarly determinate, nor is it to say that the pre-theoretic notion (or notions) exactly matched the formal definition. (Shapiro 2013, p. 168)

I will return to this point about the relationship between everyday mathematical concepts and their set-theoretic counterparts later in section 5 .

Let's note that Schlimm (Schlimm 2012) and Bartha (Bartha 2010) also discuss Lakatos in terms connected to Waismann's notion of open texture. Schlimm's central thesis is that we should be pluralists about mathematical concepts, accepting both Fregean and Lakatosian concepts in mathematics. These are summarised as follows:

According to the first, concepts are definite and fixed; in contrast, according to the second notion they are open and subject to modifications. (Schlimm 2012, p. 128)

The difference here clearly matches the difference as I have been deploying it between Lakatos's dynamic, dialectical approach and the more traditional, static approach. The characterisation of Lakatosian concepts is as "subject to modifications" and "fluid" (Schlimm 2012, p. 43), which is not quite open texture by either of the definitions we have seen above. However, Schlimm does discuss the term 'open-textured' with reference to Bartha's work and Bartha, in turn, applies the framework explicitly to Lakatos:

Very similar observations apply to mathematics. There are opentextured mathematical concepts. Lakatos (1976) famously reconstructs the reasoning that leads us to include or exclude certain objects from the category of regular polyhedra. Here, too, opentextured concepts are the ones under investigation. (Bartha 2010, p. 10)

Bartha's ultimate point is one about the role of analogy in reasoning and argumentation, which we may set aside. Nonetheless, this is another recognition of the close proximity between Waismann's open texture and Lakatos's ideas on mathematical concepts.

\section{On Safe Spaces for Mathematical Concepts}

Now that we have seen that mathematical concepts can be open-textured, this paves the way for us to apply the insights from conceptual engineering. Having argued the case that mathematical concepts may be dynamic and open to revision and development, we can draw on the tools of conceptual engineering to guide and evaluate this process. In this section, I will be applying a particular aspect of Cappelen's account of conceptual engineering to investigate the extent to which we are in control of the mathematical concepts we want to be engineering. 
In (Cappelen forthcoming, Chapter 7), Cappelen argues that the processes involved in conceptual engineering are inscrutable and out of control. In particular, "the detailed mechanisms that underpin particular instances of conceptual engineering are too complex, messy, non-systematic, amorphous, and unstable for us to fully grasp or understand" (Cappelen forthcoming, Section 7.1) and that hereby we would be mistaken to think that any individual or group has the power to control the development of concepts in any substantial way. An important corollary of these that Cappelen argues for is that there are no safe spaces where we are in control of the concepts we use or the meanings of our terms. ${ }^{7}$ Cappelen points to the large number of phenomena that affect meanings that have been catalogued in his book, not just intentional conceptual engineering but also semantic drift, semantic plasticity, messy stipulations, expert opinions, scientific reclassifications and explications, etc. All of these can lead to flux or uncertainty in the concepts we possess in ways that cannot be accurately predicted. To the list we can add the open texture of concepts: if concepts are only properly delimited for a specific domain of application, this does not point to us being in control of them in general. ${ }^{8}$

Such a conclusion does contrast with what Blackburn had in mind for the process of conceptual engineering, when deploying one of the early uses of the term:

I would prefer to introduce myself as doing conceptual engineering. For just as the engineer studies the structure of material things, so the philosopher studies the structure of thought. Understanding the structure involves seeing how parts function and how they interconnect. It means knowing what would happen for better or worse if changes were made. This is what we aim at when we investigate the structures that shape our view of the world. Our concepts or ideas form the mental housing in which we live. We may end up proud of the structures we have built. Or we may believe that they need dismantling and starting afresh. But first, we have to know what they are. (Blackburn 1999, p. 1)

Cappelen's arguments, as well as the ubiquitous presence of open texture in our concepts, dispels the idea that we can first analyse what our concepts are and only then begin with a project of conceptual engineering. The moral is that if we wait to have definite control, or to have established a safe space, then we will never even begin the second stage of taking an active role in our dynamic

\footnotetext{
${ }^{7}$ An interesting observation is how reminiscent Cappelen's introduction of the lack of safe spaces is to Lakatos's assertion that the dogmatist constantly retreats to safer ground in response to sceptical arguments. Compare "In the case of conceptual engineering, it often takes the form of the idea that there are safe spaces we can retreat to where we really do have control over the meaning of our terms." (Cappelen forthcoming, Section 7.4) to the introduction to (Lakatos 1976).

${ }^{8}$ It is not clear that this works as well with OT2, since the freedom of rational agents to go either way does not directly link to a lack of control in language. On the other hand, that version of open texture is used by Shapiro to give an account of vagueness, which is a better indication of the difficulties of natural language.
} 
conceptual repertoire. This links to Cappelen's third principle, that despite the messiness of conceptual engineering we should keep trying and not be deterred in our efforts to improve and refine our concepts.

In establishing that there are no safe spaces for concepts where we do have control over the meanings of our terms, Cappelen addresses a potential counterexample that such spaces do exist. In particular, in a legal setting, it appears that judges often do take control over how important terms are to be interpreted, and what falls in the extension of a particular term. The toy example (simplified from reality) he gives is of courts interpreting 'person' to include corporations in its extension. If this is right, the objection goes, then there are instances where we can take control of our concepts and so the 'Lack of Control' picture Cappelen has given is wrong.

Cappelen responds that this is the incorrect interpretation of what is going on in such a case. It is not that the courts have been able to control the meaning of the term 'person', but rather that a court has sufficient power to force people to misinterpret a particular term

What has happened is that they've made/forced certain people to misinterpret sentences containing 'person'. The US supreme court or any other group can no more change the meaning of 'person' than I can. They can make proposals. The difference between the legal system and me is that they have police power to back up their proposals - so even if it's an idiotic proposal, they can force people to act as if it were true. If I had a private army I could do the same to a group of people: I could get them to act as if 'pig' means 'dog', but that doesn't change the meaning of 'pig'. (Cappelen forthcoming, Section 7.4)

This seems all well and good for the case at hand. However, I would now like to turn to another potential counterexample of a safe space where there is the strong inclination that we do have control over meanings or concepts: mathematics.

In the case of mathematics, there is a standard methodology for introducing new concepts and defining how new terms are to be used. Definitions are made explicitly, and in terms of known concepts, terminology and notation. The longrunning project of ensuring rigour in mathematics means that mathematicians are in general careful to make their definitions as precise and perspicuous as possible. For example, consider the definition of a metric space:

Definition. A metric space is an ordered pair $(S, d)$ such that $S$ is a set and $d$ $i s$ a distance function on that set, where for all $x, y, z \in S$ :

1. $d(x, y) \geq 0$.

2. $d(x, y)=0$ iff $x=y$.

3. $d(x, y)=d(y, x)$.

4. $d(x, z) \leq d(x, y)+d(y, z)$. 
The notions this relies on are well-known ones, and the definition is explicit in telling us what properties the distance function needs to have. It can be argued, then, that the many phenomena that make regular terms and concepts hard to pin down are not going to show up in the tightly-controlled space of mathematics. Mathematical terms can be defined with precision and their meanings communicated clearly. There are some obvious controls on this, such as consistency with standard terminology, which mean that we still don't have complete freedom. For example, we are not free to define existing terms in totally unrelated ways (so as to avoid unwanted ambiguity). Nonetheless, mathematics can be argued in this way to be a conceptual safe space where we do have a great deal of control to introduce new terminology and decide what it means.

The difference between the mathematical realm and Cappelen's preferred examples is that the community of mathematicians is significantly smaller than the more general communities using everyday concepts or terms. Cappelen states that a philosophy paper does not have the power to enforce their definition of 'intuition', for example, since

Saying you want a word to mean something doesn't make it so. What you say, using that word, is governed by what the word means, not by what you said it meant. (Cappelen forthcoming, Section 7.4)

In contrast, following only a handful of conventions, a mathematician can and regularly does introduce new concepts and terms. If someone were to insist that a metric space doesn't satisfy the triangle inequality (the last property on the definition) then I have the weight of the mathematical community behind me to say they are wrong. Conversely, I could define a new structure that drops the triangle inequality and call it a 'schmetric space', and it would be well-defined and treatable in the standard mathematical ways. Such a switch, though, does mean that the subject is changed. Importantly, what words mean is protected in mathematics, and the freedom one has to define terms is well-regulated. The size of the community is important because everyday concepts can be interrupted by all kinds of phenomena due to the massively shared ownership of language, but in mathematics, part of being a mathematicians is that one is using the core concepts in the right way and the right way involves the strict, explicit definitions.

However, I don't believe this is quite correct. While the argument does show that there is an unusually high level of control for mathematics, in line with the level of precision and rigour often required in the discipline, this does not result in mathematics being a safe space where we have full control. The picture of mathematics as being entirely free from the normal complexities of language is an illusion. Let me argue this point now.

First of all, we can bring to bear the above considerations of open texture in mathematics. Shapiro brought out the point from Lakatos that mathematical concepts can be open textured and that an important part of the development and evolution of mathematical concepts involves responding to new ideas and questions about them. The dialogue in Proofs \& Refutations provides examples where a definition is seemingly robust, only to find that it fails to be clear for 
a range of cases. No individual in the dialogue has control over the concepts they are discussing (polyhedron, edge, vertex, face etc.), nor do they collectively have the power to establish the meanings of their terms due to the high level of disagreement and their differing prior notions of what does and does not fall under the concept of polyhedron. This paints the opposite picture: that mathematics is far from a safe space after all. The dialectical account of mathematics that Lakatos offers rests on the idea that concepts have the flexibility to change through our practices, and this flexibility stands directly against the strong picture of security above.

A standard move in the literature is to separate between the context of discovery and the context of justification. The idea is that mathematics is separated into a realm of intuitive and informal mathematics for creative thinking and discovery, and a formal and rigorous realm for ensuring results are fully justified. As Hersh puts it (using the metaphor of a "frontstage" and "backstage"):

Compared to "backstage" mathematics, "front" mathematics is formal, precise, ordered and abstract. It is separated clearly into definitions, theorems, and remarks. To every question there is an answer, or at least, a conspicuous label: "open question". [...] Compared to "front" mathematics, mathematics "in back" is fragmentary, informal, intuitive, tentative. We try this or that, we say "maybe" or "it looks like". (Hersh 1991, p. 128)

It could then be argued that the Lakatosian point is limited to the context of discovery and that the safe space of mathematics is by definition carefully curated, so must refer only to the context of justification and thereby exclude the phenomena like open texture. However, this can be interpreted as another retreat and a shrinking of the safe space to only include mathematics in the context of justification. Even then, though, there is a great deal to say against this having identified a conceptual safe haven. Lakatos himself is rejecting the 'Euclidean' ideal of there being some secure safe space that can be set apart from how mathematics is discovered, though evaluating this aspect of his programme would take us too far afield. The salient point can be made more easily, though. Consider Raman-Sundström's analysis of the history of the mathematical concept of compactness in (Raman-Sundström 2015). She examines how different notions of compactness evolved and developed over time, and contrasts differing proposed definitions. Her conclusion tells us:

The story of how open-cover compactness came to be seen as the right one is a story of developing mathematics without always knowing where it was going, how important terms should be defined, and how widely they might be applied. (Raman-Sundström 2015, p. 633)

Crucially, the various concepts of compactness are all given standard mathematical definitions, up to the same standard as 'metric space' above. Much of the work done on these would fall into the kind of published work that would 
fall under the context of justification, if such a distinction can be made substantial. Nonetheless, Raman-Sundström's discussion shows that this did not hinder the fact that the concept of compactness was evolving and developing, in line with the above arguments for the open texture of mathematics. Note also that what is considered the 'right' definition is only with respect to current practice; the open texture for mathematical concepts would suggest that this will not necessarily remain so.

A further response to mathematics being a safe space immune to the vagueness and complexity of natural language phenomena is that this is demonstrably not so. For example, there is the issue of semantic contamination, where terms which have both a natural language and a mathematical meaning have their natural language meanings affect how they are understood when they are being used in mathematics. Several studies have been carried out into the issue of semantic contamination in mathematics and these show that this does regularly occur (Tall \& Vinner 1981; Pimm 1987; Monaghan 1991; Schleppegrell 2007; Mejía-Ramos \& Inglis 2011). For instance, Monaghan's examples concern terms relating to convergence, such as 'tends to', 'approaches', 'limit' and 'converges', and he demonstrates that students let their natural language understanding of these terms affect how they understand the mathematical terms. Meanwhile, Mejía-Ramos \& Inglis show that students respond differently to mathematical argument evaluation tasks depending on whether mathematical proof is referred to in noun or verb form (i.e. the difference between "give a proof that" and "prove that"). They show that the noun version is more readily associated with an understanding of proof as a valid argument, whereas the verb version connected more closely to an argument which is convincing. As another example, a similar effect of semantic contamination was observed in (Martin \& Pease 2013) in their study of online mathematical collaboration in the third mini-polymath. The problem being considered involved a line rotating around various points in the plane, which participants termed a 'windmill'. However, Martin \& Pease observe:

In the early stages of understanding the problem, a number of participants were misled by the use of the term "windmill" to think of the rotating line as a half-line, a misunderstanding that led to counterexamples to the result they were asked to prove. (Martin \& Pease 2013, p. 109)

This occurred despite the problem being set out clearly in the usual mathematical style. Once again, mathematics does not seem to be isolated from the messy phenomena of natural language.

The point I am arguing is that semantic contamination in mathematics can be used to show that mathematics is not a conceptual safe space where there is full control of concepts and meanings of terms, so despite appearances mathematics does not provide a counterexample to Cappelen's claims. However, there is a potential objection to be had here: the cases I have examined of semantic contamination are explicitly studies of students' understanding, or found in 
early stages of getting to grips with a problem such as in the case of the 'windmill', but this does not provide any material against the claim that professional mathematicians can definitively control their concepts without such confusions providing any systematic conceptual problems or terminological ambiguities. In the next section, I will argue that even in research mathematics these problems will manifest themselves.

\section{Haslanger's Manifest and Operative Concept Distinction Applied to Mathematics}

Haslanger's ameliorative project is one of the main cases of conceptual engineering, in that constructing new concepts is shown to be part of the process of bringing about social justice. For now, though, I want to pick out just one distinction which Haslanger makes frequent use of, such as in (Haslanger 2006) and (Haslanger 2012): the distinction between manifest and operative concepts. The insight underlying it is that the concepts that we deploy in our practices do not always align with the concepts we take ourselves to be deploying. The manifest concept is that which we take ourselves to be using or working with, while the operative concept is that which we are actually putting into practice.

There are two main purposes to using Haslanger's distinction. The first is to support the argument of the previous section: that mathematics does not provide a safe space for concepts, even when restricted to the just the context of justification and professional mathematicians. The second is to illustrate the broader idea that I am pushing for in this paper: that the resources of projects in conceptual engineering can and should be applied to the case of mathematical concepts.

Many examples are used to give substance to it actually being rather common in practice for manifest and operative concepts to part ways. In (Haslanger 2005) the example is of what counts as being late to school (or 'tardy'), with the difference between the official school policy and its practical implementation providing two different concepts. In (Haslanger 2006, pp. 99-100), Haslanger uses the example of what counts as a parent, with the concept splitting between a manifest concept being the biological parents, and the operative concept of primary care-giver. The intended application, though, is to the concepts related to social justice:

[W]e debunk the idea of Woman's Nature and find two concepts at work: The manifest concept of Woman's Nature - understood as defining what women are by nature in traditional terms - is an illusion; the operative concept being masked by it is constitutively constructed in terms of men's (socially conditioned) sexual responses. (Haslanger 2012, pp. 93-94)

I will now make use of this distinction in examining the concepts of mathematics. $^{9}$

${ }^{9}$ There are further complexities involved in drawing this distinction, such as how it relates 
We might think that the relationship between informal mathematical concepts and formalisations thereof follows the manifest versus operative distinction in the following way. Formal definitions and definitions in axiomatic systems can be seen as useful for a number of reasons, such as their relative exactness, investigating foundations for mathematics, unifying systems, computational mathematics, metamathematics etc. The work of figures such as Frege, Russell, Whitehead and Hilbert (plus many others besides) demonstrated that largescale formalisation was possible. A favourite point of Azzouni's is to emphasise the widespread success of Frege's project and that of Russell \& Whitehead's Principia Mathematica in their translational endeavours, contrary to popular opinion e.g. (Azzouni 2005, p. 19) and (Azzouni 2009, p. 10). The point being that the failure of naïve comprehension does not take away from much of the successful formalisation that went on. As such, it is not surprising to find that it is commonly held that these are ultimately what mathematical concepts pick out. On the other hand, the way we learn mathematics is very much about being inducted into particular mathematical practices, which lead more naturally to the informal concepts. As such, the operative concepts which we learn through mathematics education do not necessarily coincide with the manifest concepts which mathematicians might take themselves to be using.

To make this more concrete, let us look at an example: set-theoretic foundations. Declarations to the effect that mathematics is at its core just set theory are ubiquitous in mathematics and philosophy of mathematics, especially as espoused by mathematicians themselves. Here are some samples (though opening up a random selection of set theory textbooks will be enough to furnish you with others):

[T] he mathematicians identify the natural numbers with the finite von Neumann ordinals. So, contrary to received wisdom, I suggest that philosophers follow mathematical practice and identify the natural numbers with the finite von Neumann ordinals. [...] Numbers are sets. (Steinhart 2002, p. 356)

and

All branches of mathematics are developed, consciously or unconsciously, in set theory or some part of it. (Levy 1979, p. 3)

and

Set theory is the foundation of mathematics. All mathematical concepts are defined in terms of the primitive notions of set and membership. In axiomatic set theory we formulate a few simple axioms

to semantic externalism. One potential problem is that externalism might indicate that there is no manifest concept after all. Haslanger discusses this at length in (Haslanger 2006) and I think her conclusions can be relied on here. In fact, it might even be that mathematics has an easier time in holding on to the distinction because the concepts I will argue below are the manifest ones of mathematics have in most cases received rigorous mathematical articulation and treatment. 
about these primitive notions [... From such axioms, all known mathematics may be derived. (Kunen 1980, p. xi)

and

All this is in stark contrast to what we now regard as the answer to the question what mathematical entities exist? The working practitioner of classical mathematics can answer the question with one word - sets. Every mathematical entity is a set and all sets are objects, some of them being infinite objects. (Clark 2009, p. 347)

and

$[\mathrm{M}]$ athematical objects (such as numbers and differentiable functions) can be defined to be certain sets. And the theorems of mathematics (such as the fundamental theorem of calculus) then can be viewed as statements about sets. (Enderton 1977, p. 10)

Or slightly more cautiously:

[A]xiomatic set theory is often viewed as a foundation of mathemat$i c s$ : it is alleged that all mathematical objects are sets, and their properties can be derived from the relatively few and elegant axioms about sets. Nothing so simple-minded can be quite true, but there is little doubt that in standard, current mathematical practice, "making a notion precise" is essentially synonymous with "defining it in set theory". Set theory is the official language of mathematics, just as mathematics is the official language of science. (Moschovakis 2006, p. vii)

While so many quotes might seem a little excessive, I want to emphasise that this is how the situation is presented by the above authors, and that such views are common. The manifest concepts of mathematics are of sets and set theory.

However, in practice most mathematicians are not working on set theory. In the Levy quote above he even says as much with the admission that the set theory supposedly underlying all mathematics might be unconscious. ${ }^{10}$ The operative concepts will vary with the situation and who is deploying them, of course, but I contend that frequently these will come apart from the manifest, set-theoretic concepts. There is a traditional exemplification of the manifest and operative concepts I have just described coming apart, that found in (Benacerraf 1965). With the excellent story of two children educated by "militant logicists" to truly believe that all mathematics boils down to set theory, Benacerraf shows how quickly problems can arise. For the children have been taught different settheoretic representations of the natural numbers: one knows the von Neumann ordinals, the other the Zermelo ordinals. There are then examples of theorems for one of the children which are false for the other, such as $3 \in 17$ or whether

\footnotetext{
${ }^{10}$ This is also reminiscent of Azzouni's claims that mathematicians don't need to be aware of underlying derivations, as discussed in (Azzouni 2004; Tanswell 2015).
} 
any $n$-membered set can be put into one-one correspondence with the set $n$ itself. Importantly for my purposes, these issues can't be resolved by asking other people:

Attempts to settle this by asking ordinary folk (who had been dealing with numbers as numbers for a long time) understandably brought only blank stares. (Benacerraf 1965, p. 54)

Why is this so understandable? Because it is common knowledge that, while the orthodox foundationalism declares that all mathematics reduces to set theory, this is simply not what is found in practice. Not just that, but the manifest concepts of the foundational picture come apart from the operative concepts in direct mathematical ways, as claims about the membership relation holding between numbers don't even make sense unless you are working directly with sets. Now, it could be argued that the two representations are not on equal footing. Steinhart, for instance, in (Steinhart 2002) argues for the unique correctness of the von Neumann representation by what is essentially monster-barring, inventing additional demands and conditions on the concept of number which would give Lakatos a field day. Even still, this does not undermine the fact that those not separately trained in set theory would be baffled about statements of the form $3 \in 17$.

To extend the example somewhat further, observe that representing numbers in set theory is a straightforward and well-known construction. But many of the claims about set-theoretic reductions talk of almost all of mathematics, which includes a huge deal more. If we consider as an example low-dimensional topology, explored excellently in (de Toffoli \& Giardino 2014), it seems likely that the mathematicians working in this area do not know any actual set-theoretic construction corresponding to their concepts, so even if they were to agree to the claim that mathematics is founded in set theory, this would not be reflected in their operative concepts. However, even if we were to find those who did happen to know how to translate the main concepts into set theory, de Toffoli \& Giardino demonstrate that the kind of operations licenced by the topological concepts are often visual, diagrammatic and manipulative (in a tactile sense)far removed from those which one would find in the setting of set theory. So the operative concepts, i.e. the concepts as employed in practice, are frequently not set-theoretical.

A potential objection to the above is that, while the ZFC set-theoretic foundations very much give the traditional idea of the concepts underlying mathematics, they are not the only option available. Other well-known options include category theory (see Landry \& Marquis (2005)), homotopy type theory (as in The Univalent Foundations Program (2013)), or indeed Quine's New Foundations (exemplified in (Rosser 1953), and discussed in (Morris 2017)). As such, there is the concern that what I have above claimed are the manifest concepts of mathematics are not unanimously agreed upon as the concepts we take ourselves to be using. With a plurality of proposed foundations there comes a plurality of choices for the manifest concepts, but that does not seem to fit with the idea that the manifest concepts are those we take ourselves to be using. However, in 
response, there are two reasons to believe that this is not so problematic for the distinction as I have applied it above. Firstly, Haslanger's distinction between manifest and operative concepts does not require unanimous agreement on the concepts we take ourselves to be using. Of course, there needs to some shared social component to the concepts in question, as Haslanger's point is one about the social construction of concepts, but this does not require the stronger condition of everyone having to be in agreement. Indeed, Haslanger explicitly says as much:

However, if one is sensitive to the possibility that in any actual circumstance there are competing meanings (often quite explicit) that structure alternative practices, then it seems worth considering a broad range of speakers, who are differently situated with respect to the phenomenon. [...] Yet we should keep in mind that "our" concept may not be univocal; in our haste to find a univocal concept we may obscure how the concept works in a complex social context. (Haslanger 2005, p. 16)

The same will apply to mathematics, where the alternative proposals bring with them alternative practices. As it stands, the ZFC framework is the dominant practice, and so the manifest/operative distinction can be applied as above, but they also provide a recipe for applying the distinction analogously for the alternative proposals. This brings us to the second point, which is that the various options still have a great deal in common in their perspectives on formalisation of mathematics. While they might disagree over which framework provides the most natural representation of various mathematical concepts, in the current state of play the manifest/operative distinction will apply to all of them.

In conclusion, we have seen that there is a separation between manifest and operative concepts in professional mathematics. This completes the argument for the claims made in the previous section: that mathematics is not a conceptual safe space, and that the messy complexities of natural language are at work here too. In making this case, I have also outlined a more general picture of the kinds of complexities involved in mathematics, involving open texture, semantic contamination and manifest/operative concept confusions.

\section{Looking Forward: Conceptual Engineering for Mathematical Concepts}

I want to now discuss what mathematics gains from the conceptual engineering perspective and some questions that need to be engaged with to see the project of applying conceptual engineering to mathematics through.

The most pressing question is: what is the purpose of bringing the tools of conceptual engineering to bear on mathematics? The answer that I have been building throughout this paper is that conceptual engineering gives us new theoretical distinctions to approach and describe the development and change 
of concepts that is occurring anyway. I have shown that discussions of open texture, safe spaces, and manifest and operative concepts apply naturally to the mathematical realm. The Lakatosian perspective put forward in Proofs \& Refutations is often seen as an isolated phenomenon, or limited to the context of discovery alone, but we have seen that there are a wide range of complexities involved in mathematical concepts. Conceptual engineering brings together the kinds of approaches that have substantial things to say about such complexities.

The previous section ended on a somewhat negative note: that there is a difference between manifest and operative concepts in mathematics which demonstrates that the conceptual safe space of mathematics isn't so safe after all. Such an accusation of conceptual confusion is a rather serious one, not made lightly. However, we should not forget the lesson from the discussion of open texture, namely that such problems don't interfere with much of the usual practice of mathematics. We saw with the definition of a metric space that there is an unusually high degree of control in the realm of mathematics. As such, Cappelen's Keep Trying mantra is particularly applicable. The restricted scope of mathematics means that efforts are more likely to succeed; one just needs to look at the mathematical richness that has come out of the foundationalist project to see the benefits.

Other important questions that will need to be addressed going forward concern the normativity of conceptual engineering and the defects in concepts we are trying to fix with it. The methodology is mainly aimed at identifying defective concepts and replacing or improving them, so we should spend time on what kinds of defects are motivating us. It could be like the concept of truth, where inconsistency is the motivating issue, because inconsistency is generally thought to be bad for mathematics. But there are other candidates too, like the indeterminacy of the concept of polyhedron. Other mathematical concepts might be defective by being uninteresting, unfruitful or over-complicated. All of these seem to be worth consideration and worth the effort to do better conceptually, including for the sake of the mathematics it can lead to. A simple example is the decision to exclude 1 from being a prime number for the sake of the prime factorisation theorem. A more complex example is the ongoing debate between advocates of a single universe of sets (Woodin 2011) and the multiverse perspective (Hamkins 2012), where each has fruitful mathematical consequences. (It is this latter case study that I will turn to next.) Assessing the shortcomings of given concepts and improving them might thus be mathematically important too.

We might also ask what contexts or domains a given mathematical concept needs to have an improvement engineered for. It may be that engineering in mathematics should be unrestricted to maintain the unity of the discipline. On the other hand, we may want to engineer new mathematical concepts only to be used in particular technical contexts, following the model of Scharp's dual replacements for truth in (Scharp 2013). He doesn't insist that the complex logical workings of his semantics for replacement concepts for truth are understood or even known by everyone who would use the concept of truth, just that they are available in case we need them. Analogously, a similar approach could 
be taken to the difference between the usual concepts of mathematics and their set-theoretic or formal representations, as was discussed above with respect to Haslanger's distinction.

Let me now return to set theory to show the benefits of using the above considerations about conceptual engineering as part of an ongoing debate in the philosophy of mathematics.

\section{The Concept of Set: One or Many?}

Thus far I have been arguing that we can benefit a great deal from applying the techniques and theoretical distinctions from conceptual engineering to mathematical concepts. In a sense, what I have been proposing is to see conceptual engineering as part and parcel of mathematics; that the history of many of the central concepts of mathematics reveals that they were developed in response to their predecessor-concepts being found to be defective in one way or another, and mathematicians intentionally developing replacements. For example, in (Scharp \& Shapiro forthcoming) they describe the replacement of the naive notion of convergence, which relies on inconsistent uses of infinitesimals, with the two distinct notions of pointwise and uniform convergence. Similarly, Raman-Sundström's history of the concept of compactness (Raman-Sundström 2015) shows that open-cover compactness replaced the earlier concept of sequential compactness because the latter was insufficiently general. Nonetheless, such historical cases may not fully convey the need for modern mathematics and philosophy of mathematics to connect to conceptual engineering.

In this section, I will propose a more active role for conceptual engineering in a very current debate in the philosophy of set theory. The debate concerns whether there is a single universe of sets or whether there are many such universes, positions respectively associated with Woodin, such as in (Woodin 2011), and Hamkins (Hamkins 2012). We call the former the Universe view and the latter the Multiverse view, although the debate is also characterised by Koellner in (Koellner 2013) as being between the pluralist and non-pluralist positions. While in the space here I cannot hope to resolve the debate, I will argue that the best hope for a resolution is through the active deployment of the tools of conceptual engineering and I shall indicate the direction that such an undertaking should go.

Let us begin with some background and an outline of the debate, though for our current purposes I will avoid getting into the wealth of technical details underlying it and will not presuppose much in the way of background.

Modern set theory broadly takes the axioms of ZFC (for Zermelo-Fraenkel set theory with the Axiom of Choice) as its basic system, where this is intended to encode the concepts of set and membership. These axioms were developed partly as an attempt at giving a consistent account of set theory, and thereby a consistent foundation for mathematics, after paradoxes rocked the earlier logicist attempt by Frege. One of guiding questions of set theory since Cantor is about the Continuum Hypothesis $(\mathrm{CH})$, which is the claim that there exists 
no infinite cardinal number strictly between the cardinality of the integers and the cardinality of the continuum. As it turns out, this is independent of the axioms of ZFC; Gödel showed in 1940 that $\mathrm{CH}$ could consistently be added to ZFC, while Cohen showed in the 1960s that we could also construct a model satisfying ZFC and the negation of $\mathrm{CH}$. The method underlying Cohen's work is called forcing, which is used to systematically transform one model of ZFC into another, and remains a central tool in set theory today, revealing many intricacies and relationships between different models of set theory. This sets the stage for the current debate.

The Universe view is that there is a single background concept of set, which is unique and absolute. On this picture, statements of mathematics and in particular set theory do have determinate truth values, being either true or false of the actual concept of set. As such, independence phenomena such as the independence of the continuum hypothesis from the axioms of ZFC, indicate that we need to further our search for additional, plausible or natural axioms which will decide such statements. The main project aimed at carrying this out is Woodin's search via the Inner Model Programme for Ultimate- $L$, which is a particular model of set theory which would have certain remarkable and surprising features which Woodin holds will indicate that this is the correct concept of set. The idea would be that adding a new axiom stating the the universe of sets $V$ is equal to Ultimate- $L$ would resolve the major questions such as the Continuum Hypothesis. As such, the central argument for the Universe view draws on mathematical results as a way to get insights into the absolute background concept of set, as described by Woodin thus:

A far stronger view [...] which I also currently hold [...], is that there must be such an axiom [that the universe of sets is Ultimate- $L$ ] and in understanding it we will understand why it is essentially unique and therefore true. Further this new axiom will in a transparent fashion both settle the classical questions of combinatorial set theory where to date independence has been the rule and explain the large cardinal hierarchy. [...] In other words, we would have come to a conception of the transfinite universe which is as clear and unambiguous as our conception of the fragment $V_{\omega}$, the universe of the finite integers.

(Woodin 2011, p. 17)

As an important corollary, whichever such universe of sets the new axiom picks out would provide a definite answer on claims such as the Continuum Hypothesis. For further details on Woodin's project, see the excellent description in (Rittberg 2015).

In contrast, the Multiverse view defended by Hamkins is that there are many diverse and different concepts of set, where each one is associated with a particular model of set theory. The central arguments for this view draw on (1) the existence of diverse and different models of set theory, and (2) the practical experience set theorists have in dealing with them. Hamkins argues that key methods in set theory, such as forcing, are best interpreted as giving ways of moving between different universes of set theory. He says: 
Our most powerful set-theoretic tools, such as forcing, ultrapowers and canonical inner models, are most naturally and directly understood as methods of constructing alternative set-theoretic universes. A large part of set theory over the past half-century has been about constructing as many different models of set theory as possible, often to exhibit precise features or to have specific relationships with other models. (Hamkins 2012, p. 3)

He argues that the ways that set theorists go about practicing set theory involve seeing themselves as moving between different models, something which can be seen even in the diagrams commonly used in set theory which show different models side-by-side. The idea of the multiverse view is to take these different models of set theory seriously, and to see each one as associated with a distinct concept of set:

The multiverse view explains our mathematical experience with these models by positing that, indeed, these alternative universes exist, just as they seem to exist, with a full mathematical existence, fully as real as the universe under the universe view. Thus, in our mathematical experience the classical set concept splinters into a diverse array of parallel set concepts, and there seems little reason to think that we have discovered more than a tiny part of the multiverse.

(Hamkins 2012, p. 4)

Finally, the perspective on the continuum hypothesis that Hamkins argues this delivers is that we won't settle it absolutely one way or the other, but instead have arrived at a deeper understanding of its place in different models of set theory, and therefore how it fits into the broader multiverse of set concepts.

I won't try to settle this debate here. What is important to recognise, though, is that the debate is explicitly about the concept of set: the Universe view holds that there is an absolute background concept of set which we are trying to pin down, while the Multiverse view is that there are many set concepts, all extensionally identified with the different models of set theory. As such, my proposal is that the way forward in this debate is to see this as a case where we must actively engage in conceptual engineering. This will be explored in the remainder of this section. The plan is to sequentially run through the relevant questions about how to apply conceptual engineering from the previous section, and examine how they work in the debate about set theory.

To begin, we can make use of the conceptual engineering terminology from above to give a fresh angle on this debate. For instance, we can observe that the richness of set theory and the many different models it has was not something anticipated by the early practitioners, and many of the major questions now would be alien to Cantor or Zermelo. This would appear to fit with seeing the concept of set as open-textured, at least prior to some of the modern developments. On the definition OT1, this may be slightly controversial because the question of which objects count as sets, and whether this answer has a unique and absolute answer, is the issue at stake in the universe vs multiverse debate. However, 
the phenomena of unanticipated questions and essential imcompleteness which affect the fundamentals of the delineation of where a concept begins and ends, as is crucial in Waismann's examples of open texture, are clearly present in the development of modern set theory. Shapiro's OT2 is more straightforwardly applicable, as Woodin and Hamkins demonstrate that competent, rational agents can disagree on the concept of set. The payoff for thinking about the concept of set in these terms is that it sees the ongoing debate as between rival descriptions of current set theory, but the openness reveals that we can take a more active role in deciding what we want the concepts to be doing for us and fix them to achieve our own ends.

The well-rehearsed story of the naive concept of set, as associated with naive comprehension and Frege's Basic Law V, tells us how Russell's paradox implies that this concept of set is inconsistent. This tale is covered by (Scharp \& Shapiro forthcoming), and is used as an example of a defective concept where a replacement strategy could be deployed. Interestingly, the more modern concept(s) of set at the centre of the current debate will (probably) not be defective in the same way. However, I believe that the debate does indicate that there is a defect of ambiguity in the concept of set, since major figures in set theory cannot agree on whether the concept picks out a single thing or infinitely many. ${ }^{11}$ Of course, this would not be so if the debate were easily resolved. However, it does not seem as though it could be, for the kind of support the two opposing positions draw on is not straightforwardly comparable: the Universe view is supported by a particular mathematical programme founded on the search for a single absolute concept of set, while the Multiverse view is supported by both the model-theoretic multitude and the practical experience of working with different models of set theory. Furthermore, both views have been mathematically fruiftul, connecting to Woodin's many results in the Inner Model Programme on one hand, and Hamkins \& Löwe's modal logic of forcing (Hamkins \& Löwe 2008) and the set-theoretic geology project (Reitz 2007) on the other. ${ }^{12}$ However, the ambiguity of the concept at the heart of the debate is deeply unsatisfying and revising or replacing such a defective concept should be a top priority.

The response that we favour as conceptual engineers is to explicitly recognise that this is a conceptual defect, and to offer better revisions or replacements which satisfy the aims we have for the concept. To do so requires an analysis of what the aims actually are for the concept of set and for set theory. Fortunately, there is work in this direction, such as in (Maddy forthcoming) where Maddy evaluates various aims of set-theoretic foundations and contrasts the success of the Universe view, different Multiverse views and Category Theory with respect to these goals. ${ }^{13}$ According to Maddy, the primary goals include: (see Maddy

\footnotetext{
${ }^{11}$ Indeed, a proper class of them.

${ }^{12}$ We should be careful to not confuse the chronology of the latter though; the modal logic of forcing and set theoretic geology were precursors to Hamkins's arguments for the Multiverse view.

${ }^{13}$ Indeed, I will happily claim Maddy's work here as part of the conceptual engineering approach to the debate. For example, she says:
}

As far as meanings and concepts go, I personally doubt there's a fact of the 
forthcoming, p. 51)

- Metamathematical Corral To "allow for meta-mathematical consideration of the whole expanse of that vast subject at once".

- Elucidation To "provide the conceptual resources and construction techniques to clarify old mathematical notions in order to take on new demands".

- Risk Assessment To provide a scale of consistency strength (such as by using the hierarchy of large cardinals.)

- Shared Standard To "serve as a benchmark of mathematical proof".

- Generous Arena To give "a framework in which the various branches of mathematics appear side-by-side, so that results, methods and resources can be pooled".

Setting out what we want from the concept(s) of set is the first stage towards engineering the mathematical concept or concepts that will best suit these ends. It also allows us to engage in debate as to the importance of the various goals that have been set out; for example, I have argued firmly against Shared Standard in (Tanswell 2015) and against Metamathematical Corral in (Tanswell 2016). Furthermore, the above considerations about the difference between manifest and operative concepts in mathematics seem to undermine Generous Arena. Nonetheless, the main point is that the assessment and evaluation of these aims will be a key step in knowing the design parameters for the ultimate concept or concepts of set that we want to end up with.

The last set of questions in applying conceptual engineering to set theory concern the range of contexts for which we need to revise or replace the concept of set. An important consideration for this comes from above: if there is a substantial manifest/operative distinction between the set-theoretic foundations and the everyday concepts of mathematics, as I argued, then the resolution of the universe/multiverse debate will have little direct importance for most of mathematics as it stands now. This might open space for the strategy of (Scharp 2013), replacing the concept of set only in the restricted setting of certain settheoretic projects, or when the goals Maddy outlined above are salient, but letting more informal notions of set continue on in wider mathematical applications. Alternatively, one could argue for a massive replacement of the concept of set across mathematical contexts to better suit the needs we have. In certain respects, this would appear to be what advocates of the alternative foundations

matter about what is or isn't part of the current meaning/concept of set, but even if there were, and even if it didn't settle $\mathrm{CH}$, we'd be perfectly free to decide that there's good reason to move on to an enhanced meaning/concept that does. (Maddy forthcoming, p. 48)

The choice between a universe approach and a multiverse approach is justified to the extent that it facilitates our set-theoretic goals. (Maddy forthcoming, p. 49) 
(such as category theory, homotopy type theory or new foundations) are aiming at.

To conclude, it is my opinion that the debate in set theory is a prime target for conceptual engineering. Despite the debate being explicitly framed in terms of concepts, the current approaches are too restricted in trying to describe the concepts used in set theory as it is currently practiced, rather than the stronger position I advocate that the debate should be settle based on what we want to achieve with our mathematical conceptual repertoire. I have discussed this debate in terms of the defectiveness of the concept of set, the aims we have in deploying it in mathematics, and the scope for revision or replacement, and I have argued that doing so is the best way out of the conceptual quagmire the debate finds itself in.

\section{Conclusion}

In the above I have made the case that there is indeed a great deal of scope for conceptual engineering in the realm of mathematics. Mathematical concepts are open textured, as is shown by Lakatos and Shapiro, with the upshot that the eternal and definite fixity of mathematical concepts is only a limited phenomenon and often illusory. As a dual-purpose example, I showed that Haslanger's distinction between manifest and operative concepts can be applied to the identification of mathematical concepts with set-theoretic analogues, demonstrating a divide between ideal and observed practice of mathematics. Furthermore, this instantiated my claim that the tools being developed as part of the wider movement of conceptual engineering can prove fruitful when applied in the philosophy of mathematics. I have made the case that this all contributes to Cappelen's argument that there are no conceptual safe spaces where we are in full and definite control, something which mathematics would have been a prime candidate to serve as an objection to. In the end, I have argued that mathematicians are a kind of conceptual engineers. I do not merely mean this as a merely descriptive claim, but additionally as a methodology that should be embraced in mathematics. I demonstrated this as applied to the debate between advocates of the Universe view and the Multiverse view of set theory, arguing that the way forward in this debate is to understand the issue of tension as being of the right kind for conceptual engineering to resolve. Drawing on the resources of conceptual engineering will allow more powerful insights into the creation and development of mathematical concepts.

\section{Acknowledgements}

Many thanks for comments, discussion and feedback to Aaron Cotnoir, Patrick Greenough, Joshua Habgood-Coote, Brendan Larvor, Øystein Linnebo, Benedikt Löwe, Ursula Martin, Ásgeir Berg Matthíasson, Colin Rittberg, Kevin Scharp, Stewart Shapiro, and an anonymous referee, as well as audiences in New Delhi, 
Sønderborg, and St Andrews.

\section{Funding}

This work was supported by the EPSRC grant for the project "The Social Machine of Mathematics" led by Prof Ursula Martin [EP/K040251/2]. This work was developed from work in my $\mathrm{PhD}$ which was supported by the Caroline Elder Scholarship and a SASP Scholarship. The trip to New Delhi to present this work was made possible by the International Association for Science and Cultural Diversity.

\section{References}

Azzouni, J. (2004a) "The Derivation-Indicator View Of Mathematical Practice", Philosophia Mathematica (III) 12, pp. 81-105.

Azzouni, J. (2005b) "Is There Still A Sense In Which Mathematics Can Have Foundations?" in Sica, G. (ed.) Essays on the Foundations of Mathematics and Logic, Milan, Polimetrica, pp. 9-47.

Azzouni, J. (2009) "Why Do Informal Proofs Conform To Formal Norms?", Foundations of Science 14, pp. 9-26.

Bartha, P. F. A. (2010) By Parallel Reasoning: The Construction and Evaluation of Analogical Arguments, New York, Oxford University Press.

Benacerraf, P. (1965) "What Numbers Could Not Be", The Philosophical Review 74, pp. $47-73$.

Blackburn, S. (1999) Think, Oxford, Oxford University Press.

Cappelen, H. (forthcoming) Fixing Language: Conceptual Engineering and the Limits of Revision, April 2017 version.

Clark, P. (2009) "Mathematical Entities" in Le Poidivin, R.; Simons, P.; McGonigal, A. \& Cameron, R. P. (eds.) The Routledge Companion to Metaphysics, Abingdon, Routledge, pp. 346-356.

de Toffoli, S. \& Giardino, V. (2014) "An Inquiry into the Practice of Proving in Low-Dimensional Topology", in Lolli, G.; Panza, M. \& Venturi, G. (eds.) From Logic to Practice: Italian Studies in the Philosophy of Mathematics, Cham, Switzerland, Springer, pp. 315-336.

Enderton, H. B. (1977) Elements of Set Theory, London, Academic Press Inc.

Feferman, S. (1978) "The Logic of Mathematical Discovery vs. The Logical Structure of Mathematics", in PSA: Proceedings of the Biennial Meeting of the Philosophy of Science Association, Volume 2: Symposia and Invited Papers, pp. 309-327. 
Hamkins, J. (2012) "The Set-Theoretic Multiverse", Review of Symbolic Logic 5, pp. $416-449$.

Hamkins, J. \& Löwe, B. (2008) "The Modal Logic of Forcing", Transactions of the American Mathematical Society 360, pp. 1793-1817.

Haslanger, S. (2000) "Gender and Race: (What) Are They? (What) Do We Want Them to Be?", Noûs 34, pp. 31-55.

Haslanger, S. (2005) "What Are We Talking About? The Semantics and Politics of Social Kinds", Hypatia 20, pp. 10-26.

Haslanger, S. (2006) "Philosophical Analysis and Social Kinds", Proceedings of the Aristotelian Society 80, pp. 89-143.

Haslanger, S. (2012) Resisting Reality: Social Construction and Social Change, Oxford, Oxford University Press.

Hersh, R. (1991) "Mathematics has a Front and a Back", Synthese 88, pp. $127-133$.

Koellner, P. (2013) "Hamkins on the Multiverse", unpublished, available online at http://logic.harvard.edu/EFI_Hamkins_Comments.pdf.

Kunen, K. (1980) Set Theory: An Introduction To Independence Proofs, Amsterdam, North Holland Publishing Company.

Lakatos, I. (1963) "Proofs and Refutations (I)-(IV)", The British Journal for the Philosophy of Science 14, pp. 1-25, 120-139, 221-245, 296-342.

Lakatos, I. (1976) Proofs And Refutation, Cambridge, Cambridge University Press.

Landry, E. \& Marquis, J. P. (2005) "Categories in Context: Historical, Foundational, and Philosophical" Philosophia Mathematica (III) 13, pp. 1-43.

Levy, A. (1979) Basic Set Theory, London, Springer-Verlag.

Linnebo, Ø. (2016) "How to Harness Basic Law V", in Carrara, M.; Arapinis, A. \& Moltmann, F. (eds.) Unity and Plurality: Logic, Philosophy, and Linguistics, Oxford, Oxford University Press, pp. 19-32.

Maddy, P. (forthcoming) "Set Theoretic Foundations", forthcoming in Caicedo, A.; Cummings, J.; Koellner, P. \& Larson, P. (eds.) Foundations of Mathematics. Essays for W. Hugh Woodin on the occasion of his 60th Birthday, American Mathematics Society Contemporary Mathematics series.

Martin, U. \& Pease, A. (2013) "Mathematical Practice, Crowdsourcing and Social Machines" in Carette, J.; Aspinall, D.; Lange, C.; Sojka, P. \& Windsteiger, W. (eds.) Intelligent Computer Mathematics, Volume 7961 of the series Lecture Notes in Computer Science, pp. 98-119. 
Mejía-Ramos, J. P. \& Inglis, M. (2010) "Semantic Contamination and Mathematical Proof: Can a Non-Proof Prove?", Journal of Mathematical Behavior 30, pp. $19-29$.

Monaghan, J. (1991) "Problems with the Language of Limits", For the Learning of Mathematics 11, pp. 20-24.

Morris, S. (2017) "The Significance of Quine's New Foundations for the Philosophy of Set Theory", The Monist 100, pp. 167-179.

Moschovakis, Y. (2006) Notes on Set Theory: Second Edition, New York, NY, Springer.

Pimm, D. (1987) Speaking Mathematically: Communication in Mathematics Classrooms, London, Routledge \& Kegan Paul.

Raman-Sundström, M. (2015) "A Pedagogical History of Compactness", The American Mathematical Monthly 122, pp. 619-635.

Reitz, J. (2007) "The Ground Axiom", The Journal of Symbolic Logic 72, pp. $1299-1317$.

Rittberg, C. J. (2015) "How Woodin Changed His Mind: New Thoughts on the Continuum Hypothesis", Archive for History of Exact Sciences 69, pp. $125-151$.

Rosser, J. B. (1953) Logic for Mathematicians, London, McGraw-Hill Book Company.

Scharp, K. (2013) Replacing Truth, Oxford, Oxford University Press.

Scharp, K. \& Shapiro, S. (forthcoming) "Revising Inconsistent Concepts" in Amour-Garb, B. (ed.) The Relevance of the Liar, Oxford, Oxford University Press.

Schleppegrell, M. J. (2007) The Linguistic Challenges of Mathematics Teaching and Learning: A Research Review, Reading \&3 Writing Quarterly 23, pp. 139159.

Schlimm, D. (2012) "Mathematical Concepts and Investigative Practice" in Feest, U. \& Steinle, F. (eds.) Scientific Concepts and Investigative Practice, Berlin, de Gruyter GmbH, pp. 127-147.

Shapiro, S. (2006) Vagueness In Context, New York, Oxford University Press.

Shapiro, S. (2006) "Computability, Proof, And Open-Texture" in Olszewski, A., Woleński, J. \& Janusz, R. (eds.) Church's Thesis after 70 Years, Frankfurt, Ontos Verlag, pp. 420-455.

Shapiro, S. (2013) "The Open Texture of Computability" in Copeland, B. J.; Posy, C. J. \& Shagrir, O. (eds.) Computability: Turing, Gödel, Church, and Beyond, Cambridge MA, MIT Press, pp. 153-181. 
Steinhart, E. (2002) "Why Numbers Are Sets", Synthese 133, pp. 343-361.

Tall, D. \& Vinner, S. (1981) "Concept Image and Concept Definition in Mathematics with particular reference to Limits and Continuity", Educational Studies in Mathematics 12, pp. 151-169.

Tanswell, F. S. (2015) "A Problem with the Dependence of Informal Proofs on Formal Proofs", Philosophia Mathematica (III) 23, pp. 295-310.

Tanswell, F. S. (2016) "Saving Proof from Paradox: Gödel's Paradox and the Inconsistency of Informal Mathematics", in Andreas, H. \& Verdée, P. (eds.) Logical Studies of Paraconsistent Reasoning in Science and Mathematics, Trends in Logic 45, Cham, Switzerland, Springer International, pp. 159-174.

Tanswell, F. S. (2016) Proof, Rigour and Informality: A Virtue Account of Mathematical Knowledge, $\mathrm{PhD}$ thesis, University of St Andrews \& University of Stirling.

The Univalent Foundations Program (2013) Homotopy Type Theory: Univalent Foundations of Mathematics, Institute for Advanced Study, https ://homotopytypetheory.org/book.

Waismann, F. (1951) "Analytic-Synthetic III", Analysis 11, pp. 49-61.

Waismann, F., (1968) "Verifiability", in Flew, A. (ed.) Logic and Language, Oxford, Basil Blackwell, pp. 118-144.

Woodin, H. (2011) "The Continuum Hypothesis, the Generic-Multiverse of Sets, and the $\Omega$-Conjecture", in Kennedy, J. \& Kossak, R. (eds.) Set Theory, Arithmetic, and Foundations of Mathematics: Theorems, Philosophies, Cambridge, Cambridge University Press, pp. 13-42. 